\title{
Aneurismas del sector distal de la arteria cerebral anterior (arteria pericallosa)
}

\author{
F. Martinez; E. Spagnuolo y A. Calvo
}

Servicio de Neurología y Neurocirugía. Hospital Maciel. Montevideo. Uruguay.

\section{Resumen}

Se analizan las características clínicas, angiográficas y quirúrgicas de 13 pacientes portadores de aneurismas de la arteria pericallosa, operados por un mismo grupo de cirujanos, dentro de un total de 18 casos.

Hubo una mortalidad del $5.5 \%$ (tomando en cuenta los 18 pacientes operados), con resultados buenos (GOS II) o excelentes (GOS I) en el $91.7 \%$.

Según la experiencia de los autores y de otros de referencia, los aneurismas de la arteria pericallosa no deberían considerarse de peor pronóstico que aquellas otras topografías dentro del sector anterior del polígono de Willis.

PALABRAS CLAVE: Hemorragia subaracnoidea. Aneurismas de arteria pericallosa.

Aneurysms of the distal anterior cerebral artery

\section{Summary}

The clinical, angiographic and surgical features of 13 (out of 18) patients harboring pericallosal aneurysms and operated on by the same group of neurosurgeons, are analyzed.

The mortality rate of the whole series was $5.5 \%$ with good (GOS II) or excellent (GOS I) recovery in the $91.7 \%$ of cases.

According to the experience of the authors pericallosal aneurysms should not be considered to have a worse prognosis than aneurysms in other locations of the anterior circulation.

KEY WORDS: Subarachnoid hemorrhage. Pericallosal aneurysms.

Recibido: 09-07-04. Aceptado: 18-10-04.

\section{Introducción}

La hemorragia subaracnoidea (HSA) es una enfermedad que afecta entre 7 y 15 de cada 100.000 habitantes por año ${ }^{5,46,47}$, con consecuencias devastadoras; tiene una alta mortalidad y un elevado porcentaje de secuelas invalidantes entre los sobrevivientes ${ }^{5,42,47}$.

La causa más frecuente de HSA es la rotura de un aneurisma intracraneano; los que se ubican en el $80 \%$ de los casos, en el sector anterior del polígono de Willis ${ }^{6,42,47,57}$. Las topografías más frecuentes son: la bifurcación de la arteria cerebral media, la arteria carótida interna en el origen de la comunicante posterior y la arteria comunicante anterior ${ }^{42,46,58,60}$. Estas topografías llegan a representar cada una, aproximadamente, entre el 25 y $30 \%$ del total ${ }^{57}$.

Los aneurismas del sector distal de la arteria cerebral anterior (ACAD) son poco frecuentes y alcanzan del $2 \%$ al $9 \%$ del total $1,13,20,30,37,46,47,58,60,62$. Esta baja frecuencia, hace que cada neurocirujano tenga una relativa poca experiencia en su manejo ${ }^{62,63}$. A esto se suman algunas dificultades técnicas en su tratamiento, como: su frecuente asociación con otros aneurismas, cuello con placas calcificadas en su base, espacio de abordaje estrecho en la cisura interhemisférica, adherencias a la circunvolución del cíngulo, dificultades en su localización durante la cirugía; y, a diferencia en cuanto a las vías habituales de abordaje, que dependen en definitiva de la localización estricta de la malformación, su dirección, relaciones con estructuras vecinas, etc. ${ }^{29,60}$.

Por todos estos factores, en general, se considera a los aneurismas de la ACAD como de difícil tratamiento y con un pronóstico peor en relación a otros aneurismas del sector anterior del polígono ${ }^{4,13,15,29,59}$. Sin embargo, trabajos más

Abreviaturas: AAPC: aneurismas de la arteria pericallosa. ACAD: arteria cerebral anterior distal. ACM: arteria callosomarginal. AFP: arteria frontopolar. APC: arteria pericallosa. GCS: Glasgow coma sacale. GOS: Glagow outcome scale. HEC: hipertensión endocraneana. HIV: hemorragia intraventricular. HSA: hemorragia subaracnoidea. HTA: hipertensión arterial. MAV: malformación arteriovenosa. PIC: presión intracraneana. WFNS: federación mundial de sociedades de neurocirugía. 
Tabla 1

Categorización de los pacientes por edad, sexo y grado en las escalas de Fisher, Hunt y Hess (H y H), WFNS y GOS. En los pacientes que cambiaron su grado en la escala de H y H y WFNS entre el ingreso y la cirugía se muestran ambos valores (ingresolpreopertorio)

\begin{tabular}{|c|c|c|c|c|c|c|}
\hline PACIENTE & SEXO & EDAD & FISHER & H y H & WFNS & GOS \\
\hline 1 & M & 61 & III & II/IV & II/IV & V \\
\hline 2 & M & 46 & III & I & II & I \\
\hline 3 & F & 48 & III & II & II & I \\
\hline 4 & F & 34 & IV & I & I & I \\
\hline 5 & M & 69 & II & II & II & I \\
\hline 6 & M & 51 & IV & I/III & I/III & II \\
\hline 7 & M & 40 & I & I & I & I \\
\hline 8 & M & 55 & III & V/IV & V/IV & II \\
\hline 9 & M & 53 & III & III & III & I \\
\hline 10 & M & 51 & IV & IV/II & IV/II & I \\
\hline 11 & M & 57 & I & II & II & I \\
\hline 12 & M & 32 & II & I & I & II \\
\hline 13 & M & 34 & III & I & I & I \\
\hline
\end{tabular}

recientes indican que la morbimortalidad en los aneurismas de la ACAD no es mayor que en los de otras topografías $8,10,17,19,37,62,63$.

En el presente trabajo se analizan las características clínicas y el tratamiento de 13 pacientes portadores de aneurismas de la ACAD tratados por un mismo grupo de cirujanos.

\section{Material y métodos}

Desde octubre de 1986 hasta abril del 2004, dos neurocirujanos (A. Calvo Rubal y E. Spagnuolo) operaron 750 aneurismas intracraneanos. De ellos, 18 (2.5\%) se situaron en la arteria pericallosa o sus ramas. Las intervenciones se llevaron a cabo en 4 centros hospitalarios de Montevideo: Hospitales Maciel, de Clínicas y Policial, y Asociación Española Primera de Socorros Mutuos.

Del total de 18, se obtuvieron las historias clínicas completas en 13 pacientes, haciéndose constar: presentación clínica del cuadro, examen físico al ingreso, resultados del estudio tomográfico y la arteriografía de los 4 vasos del cuello; junto con el estado clínico preoperatorio, abordaje quirúrgico utilizado y último control en policlínica.

Los datos de las 5 historias restantes son incompletos, a pesar de poseerse una detallada descripción operatoria, por lo que no se incluyen dichos casos en el análisis, aunque se sabe que sobrevivieron sin contratiempos a la intervención.

Para determinar el estado clínico preoperatorio se utilizaron las escalas de Hunt y Hess y de la WFNS. Para valorar los hallazgos tomográficos se utilizó la escala de Fisher. El estado clínico al alta y en el último control en policlínica, se cuantificó según la Glasgow Outcome Scale (GOS).

\section{Resultados}

\section{Características de la población en estudio}

De los 13 pacientes, 2 fueron mujeres y 11 hombres, lo que muestra una relación de 5:1. Las edades estuvieron comprendidas entre los 32 y 69 años, con un promedio de 48.6.

Entre los antecedentes personales de los pacientes, se hallaron: 5 casos de hipertensión arterial (HTA), 4 individuos tabaquistas; alcoholismo y obesidad en una oportunidad respectivamente.

Dos pacientes tenían antecedentes familiares de enfermedades cerebrovasculares; uno de ellos con un hermano portador de una malformación arteriovenosa (MAV) y un primo fallecido por HSA; otro, con un hermano fallecido por HSA. Este paciente es el más joven de la serie. 
Tabla 2

Presentación clínica

\begin{tabular}{|l|c|}
\hline SINTOMA & NUMERO DE CASOS \\
\hline Cefalea & 11 \\
\hline Vómitos & 7 \\
\hline Pérdida de conocimiento & 4 \\
\hline Déficit motor & 4 \\
\hline Crisis & 3 \\
\hline Fotofobia & 3 \\
\hline Coma - Depresión del sensorio & 2 \\
\hline
\end{tabular}

\section{Presentación clínica (Tablas 1 y 2)}

Los síntomas más comunes fueron los relacionados con la hipertensión endocraneana (HEC). Una cefalea brusca, "explosiva", característica de una HSA, se presentó en 11 oportunidades. Fueron menos frecuentes los síntomas motores deficitarios, crisis epilépticas y depresión de la conciencia. Dentro de los pacientes con déficit motor $(n=4)$, dos presentaron una paraparesia, otro una hemiparesia de predomino crural contralateral al aneurisma.

El cuarto paciente ingresó con una triparesia (miembro superior derecho y ambos miembros inferiores) y agregó una cuadriparesia en la evolución preoperatoria, que posteriormente mejoró.

En el examen físico, en el 83\% de los pacientes (11/13) se comprobó una rigidez de nuca; dentro de los que no la presentaron, uno tenía un examen físico normal luego de una crisis tónico-clónica generalizada, el otro entró en coma bruscamente.

En cuanto al estado clínico del ingreso, hubo una correlación casi exacta entre las escalas de Hunt y Hess y de la WFNS. De los 13 sujetos, 6 mostraron una HSA grado I, 4 un grado II y se encontró 1 caso de los grados III, IV y V, respectivamente.

En la evolución, cuatro enfermos cambiaron su grado: dos mejoraron y dos se agravaron. De estos, uno falleció en el postoperatorio.

\section{Número de consultas}

De los 13 pacientes, 12 fueron diagnosticados como portadores de HSA en la primera consulta. El caso restante, solicitó atención tres veces y recién en la última se pensó en HSA. En la primera consulta, se diagnosticó patología digestiva, ya que ingresó por cefaleas y vómitos. En la segunda oportunidad, se planteó una crisis conversiva,
Tabla 3

Hallazgos tomográficos al ingreso

\begin{tabular}{|lc|}
\hline HALLAZGO TAC & NUMERO DE CASOS \\
\hline Hemocisterna interhemisférica & 5 \\
\hline HSA holocisternal & 6 \\
\hline Hidrocefalia & 3 \\
\hline HIV & 2 \\
\hline Hematoma frontal & 1 \\
\hline Normal & 3 \\
\hline
\end{tabular}

luego de que el paciente tuviera una crisis tonicoclónica generalizada. Posteriormente, repitió la crisis, siendo trasladado a la emergencia de un Hospital terciario (Hospital Maciel), donde se hizo una TAC de cráneo que llevó al diagnóstico de HSA.

\section{Hallazgos tomográficos (tabla 3)}

En todos los casos se hizo una tomografía axial computada (TAC) de cráneo al ingreso. La misma, permitió el diagnóstico de HSA en 9 oportunidades.

En dos pacientes los hallazgos fueron: una zona de isquemia en el territorio de la arteria frontopolar, con realce postcontraste a nivel interhemisférico anterior, lo que hizo sospechar la malformación vascular. En otra oportunidad, se halló un hematoma frontal en una mujer de 34 años sin antecedentes a destacar.

En los 2 casos restantes (16.7\%), el diagnóstico se hizo mediante punción lumbar, luego de una TAC normal y un cuadro clínico altamente sugestivo de HSA.

\section{Situación y morfología de los aneurismas en la ACAD}

De los pacientes de esta serie, 12 (91.7\%) tuvieron aneurismas saculares y uno era un aneurisma fusiforme. Este último presentaba un sector trombosado, por lo que, a la vista de la imagen, fue interpretado como sacular, haciéndose el diagnóstico de la variedad, en el intraoperatorio.

Ocho aneurismas se situaron en la unión de las arterias pericallosa-callosomarginal, 5 en la unión pericallosa-frontopolar (APC-AFP). En tres oportunidades, un aneurisma de la unión APC-AFP fue interpretado radiológicamente como un aneurisma de la arteria comunicante anterior y abordado por un colgajo pterional, llegándose al diagnóstico preciso de su topografía, durante la intervención.

Cinco individuos fueron portadores de aneurismas múltiples: dos asociaron un aneurisma de comunicante 
anterior, otro un silviano homolateral, otro un carótidooftálmico homolateral y el restante un aneurisma de la bifurcación carotídea contralateral.

\section{Abordaje quirúrgico y "timing"}

Los pacientes de esta serie fueron operados por dos tipos de abordaje: pterional (6 casos) y precoronal interhemisférico (7 casos). La elección del mismo se basó en la topografía del aneurisma y la presencia o no de aneurismas múltiples.

Los aneurismas pericallosos cercanos a la arteria comunicante anterior, se abordaron por pterional. Salvo uno, los casos de aneurismas múltiples se abordaron por pterional, con la ampliación necesaria si el aneurisma pericalloso se situaba muy distal a la arteria comunicante anterior. En el caso restante, el responsable del sangrado por la TC había sido el aneurisma de la bifurcación carotídea contralateral. Se intervino en agudo éste, y el pericalloso contralateral y que era distal, se intervino en diferido por un colgajo precoronal interhemisférico.

El tiempo transcurrido entre el inicio del cuadro y la cirugía fue entre 3 y 99 días (promedio 29.1 días). Un paciente (caso 7), se operó en el día 99 debido a que ingresó evolucionado, era fumador, obeso y portador de una HTA de difícil control. Esto dificultó su preparación para la cirugía; fue coordinado en tres oportunidades y se suspendió por tener cifras sistólicas de $260 \mathrm{~mm} \mathrm{Hg}$. en el preoperatorio inmediato.

Si este paciente no se incluyese, por lo excepcional de su evolución preoperatoria, el promedio de días de espera sería de 22.1. Sólo un paciente fue operado en fase aguda.

\section{Evolución postoperatoria}

Los resultados postoperatorios se valoraron según la GOS. En 12 pacientes fueron muy buenos o buenos: GOS I y II en 9 y 3 casos respectivamente. Los GOS I están completamente reintegrados a su vida normal, salvo un paciente que falleció dos años después de la cirugía, por un linfoma no Hodking. Los 3 casos con GOS II están parcialmente reintegrados a sus actividades, con ciertos trastornos neuropsíquicos, pero sin déficit motor.

Un enfermo falleció (GOS V, caso 1). Esto hace que la mortalidad en los casos completamente estudiados sea del $7.7 \%$, pero teniendo en cuenta los 18 pacientes operados, en realidad sólo uno falleció (5.5 \%). Hay que destacar que este sujeto presentaba además un aneurisma de la arteria cerebral media, que había sido el responsable del sangrado. El paciente se complicó con un vasoespasmo e infarto en el territorio de dicha arteria, responsable en definitiva, del desenlace fatal.

Por lo tanto, en lo que se refiere estrictamente a los aneurismas de la arteria pericallosa, ningún paciente falle-
2005; 16: 333-344

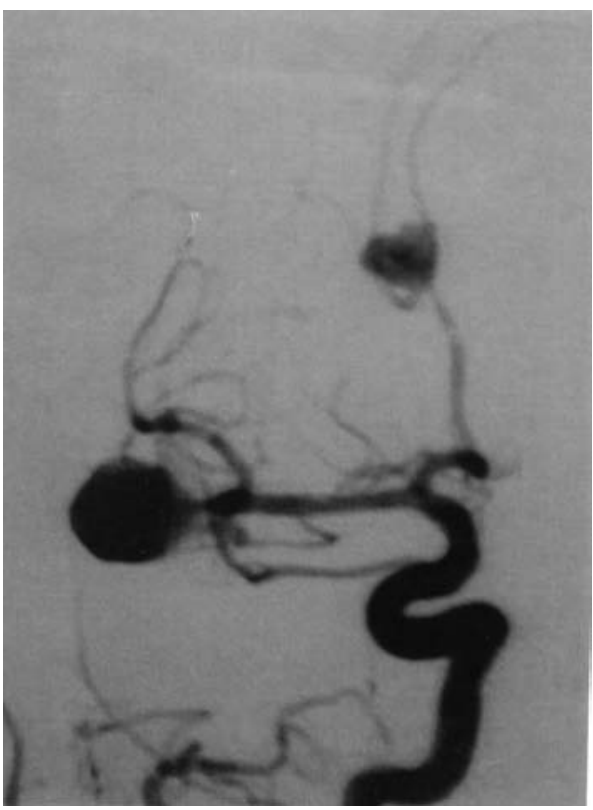

Figura 1. Arteriografia carotidea derecha. Enfoque de frente. Se observan dos aneurismas, uno en bifurcación silviana y el otro en la arteria pericallosa. Obsérvese que este último se situa en la bifurcación pericallosa-callosomarginal.

ció por la cirugía de los aneurismas de esta topografía.

\section{Casos clínicos representativos}

Caso 1: SF, 61 años, fumador e hipertenso. Ingresó por cuadro de cefaleas, fotofobia y vómitos de inicio brusco. En emergencia estaba vigil, parcialmente orientado, cumpliendo órdenes (GCS 14). Al examen se constató rigidez de nuca y PA de 200/120. La TAC de cráneo evidenció HSA holocisternal supra e infratentorial, con hidrocefalia leve (Hunt y Hess II, Fischer III). A los tres días deprime la conciencia, llegando a GCS 9 y agrega paresia de hemicuerpo izquierdo. La TAC de control no mostró aumento de la hidrocefalia ni isquemia configurada. La radiografía de tórax evidenció neumonía basal derecha, por lo que requirió intubación orotraqueal e ingreso desde la Unidad de Cuidados Intermedios al Centro de Tratamiento Intensivo (CTI).

La arteriografía reveló: aneurisma de la arteria cerebral media y de la arteria pericallosa a derecha (figura 1). Se operó el día 13 (Hunt y Hess III), clipándose ambos aneurismas por abordaje pterional. Mala evolución posterior con PIC altas y GCS 7.

Tomografía de control: infarto en el territorio de la arteria cerebral media derecha con efecto de masa. La evolución fue mala, con hemodinamia inestable. Presentó complicaciones extraneurológicas con traqueobronquitis 


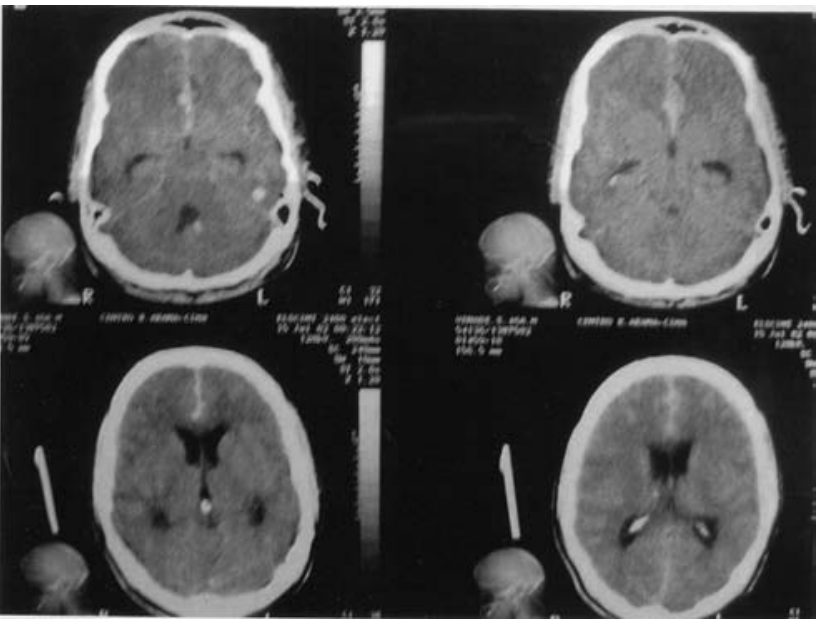

Figura 2. Tomografía axial computarizada de cráneo. Se evidencia una hemorragia subaracnoidea a predominio en la cisterna interhemisférica anterior.

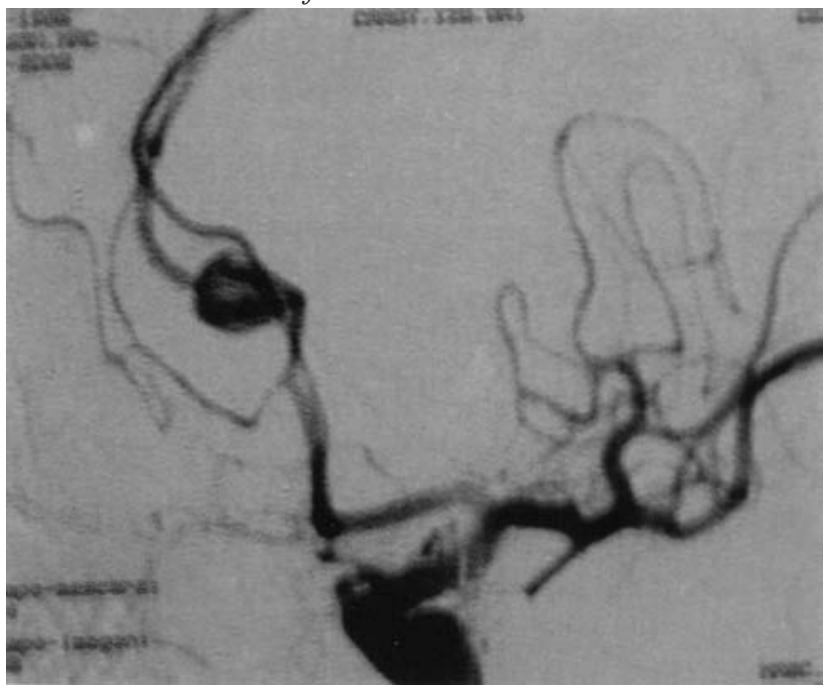

Figura 3. Arteriografía carotídea izquierda. Enfoque oblicuo anterior izquierdo. Se observa el aneurisma pericalloso situado algo distal a la arteria callosomarginal. Si bien este anuerisma aparenta ser sacular, en el intraoperatorio se vio que era fusiforme y presentaba un sector trombosado.

purulenta y atelectasia basal derecha. Empeoramiento clínico, GCS 3, con pupilas fijas y fallece en el tercer día del postoperatorio.

Caso 2: J V, sexo masculino, 46 años. AF: hermano con MAV y primo fallecido por HSA.

El 13-7-02 presenta pérdida de conocimiento, interpretada como crisis conversiva. A los dos días vuelve a consulta por dolor abdominal y vómitos, por lo que se infiere estar frente a un cólico hepático. En la noche del mismo día, tiene una pérdida de conocimiento y posteriormente queda confuso. Es trasladado a Emergencia del Hospital Maciel

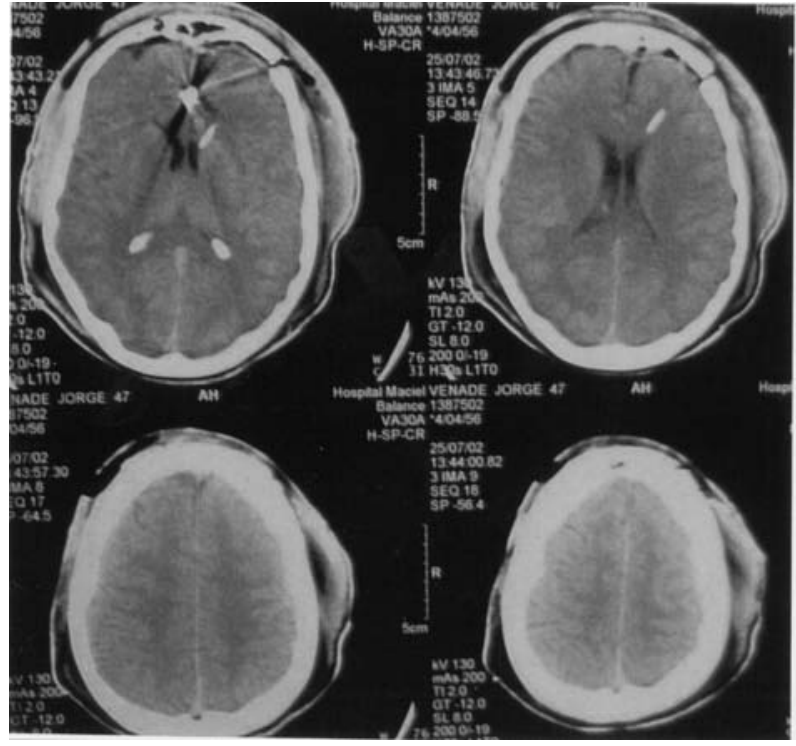

Figua 4. Tomografía axial computarizada de cráneo, control postoperatorio. En este estudio se ve el clip metálico en la cisterna interhemisférica anterior y un catéter ventricular (derivación externa). No hay complicaciones postoperatorias.

donde ingresa con cefaleas. Al examen, sólo se constata rigidez de nuca. Se hace TAC que evidencia HSA en la cisura interhemisférica anterior (figura 2). H y H I, Fischer III.

La arteriografía de los 4 vasos del cuello reveló un aneurisma de la APC izquierda (figura 3). Se interviene el 17-7-02 por abordaje interhemisférico precoronal izquierdo (abordaje bicoronal en piel). Se hizo disección próximo-distal, hallándose aneurisma fusiforme. Se coloca un clip recto a la derecha del aneurisma y se coagula el sector izquierdo sin compromiso del vaso madre (figura 4).

Buena evolución posterior. El paciente es dado de alta a la semana sin déficit, GOS I. En el último control en policlínica en setiembre de 2003, se encuentra asintomático y completamente reintegrado a sus actividades habituales como cartero.

Caso 7: J. O., 40 años, sexo masculino. AP: hipertenso, fumador de 30 cigarrillos diarios, obeso, alcohólico.

El 13-05-02 sufre crisis tónico-ciónica generalizada con pérdida del control esfinteriano. Es visto por una emergencia médica móvil, que lo traslada a la puerta de Hospital Maciel.

Al examen del ingreso se constata: vigil, orientado, cumple órdenes; examen neurológico normal.

Se hace TAC de cráneo que muestra una imagen hipodensa en el territorio de la arteria frontopolar derecha, con un sector parasagital que capta contraste, planteándose una malformación vascular.

Con diagnóstico de crisis epiléptica y lesión frontal, probablemente secundaria a un hematoma frontal 


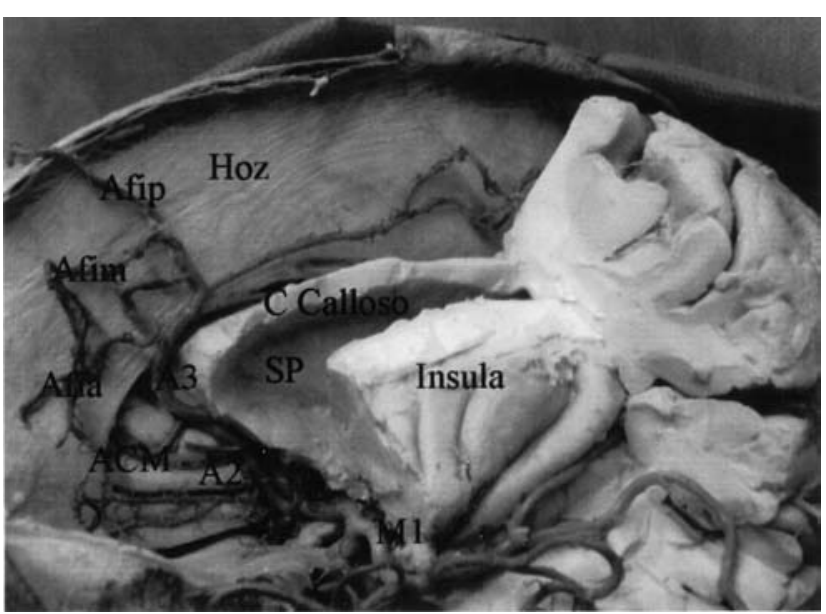

Figura 5. Pieza anatómica, vista lateral del hemisferio cerebral izquierdo. Se han resecado el lóbulo frontal $y$ parte de los lóbulos temporal y parietal para poner en evidencia el trayecto de la arteria pericallosa. Se observa la bifurcación carotídea y el inicio de las arterias cerebrales anterior y media (M1). Distal con respecto a la arteria comunicante anterior, se ve el sector vertical de la callosa (A2 y A3). Posteriormente, contornea el cuerpo calloso (c calloso) quedando por debajo de la hoz del cerebro (hoz). Se observa claramente el origen de la arteria callosomarginal (ACM) del sector vertical y un tronco común entre las tres arterias frontales internas (Afia, Afim y Afip). Por debajo del cuerpo calloso se ubica el septum lucidum (SP), con la vena septal anterior.

parasagital derecho pequeño (a pesar de no haber noción clínica de sangrado previo), y sospecha de aneurisma por la TAC, se decide completar el estudio con una angiografía de los cuatro vasos del cuello.

La misma puso en evidencia un aneurisma de la arteria pericallosa derecha, de $8 \mathrm{~mm}$ de diámetro máximo y $6 \mathrm{~mm}$ de diámetro transversal, con un cuello de $5 \mathrm{~mm}$. El fondo estaba dirigido hacia arriba, adelante y algo a la izquierda.

Se coordinó en tres oportunidades para cirugía, y se suspendió otras tantas por cifras tensionales elevadas, de hasta $260 \mathrm{~mm} \mathrm{Hg}$ de sistólica. Se opera el 22-8-02 por colgajo paramediano precoronal derecho. Se aborda por interhemisférico, con disección de distal a proximal. Se ubica el aneurisma en la bifurcación APC-ACM, clipándose sin incidentes. En el intraoperatorio se identifica el área frontopolar homolateral comprobándose un sangrado previo, ya que la zona tenía coloración ocre. Buena evolución, pasa en Cuidados Intensivos dos días, y es dado de alta de sala el 27-8 con GOS I. En el último control (noviembre de 2003) está asintomático y reintegrado a sus tareas habituales

\section{Discusión}

Los aneurismas de la arteria pericallosa son relativamente poco frecuentes, y representan entre el $1.5 \mathrm{y}$ el $9.2 \%$ del total de los aneurismas intracraneales ${ }^{6,14,28,30,54,57}$. Clásicamente se considera que, dentro de los aneurismas, los del sector anterior, tienen peor pronóstico que los de otras topografías. Sin embargo, trabajos recientes ponen en evidencia que los resultados de la cirugía en estos aneurismas no es significativamente diferente al resto de los aneurismas de la circulación anterior ${ }^{5,18,21,29,37,46}$.

\section{Anatomía quirúrgica de la ACAD (figura 5)}

La arteria cerebral anterior (ACA) es la rama terminal anterior de la arteria carótida interna ${ }^{23,35,40,42,50}$. Desde su origen en el sector más interno de la cisura de Silvio, se dirige hacia delante y adentro cruzando por arriba al quiasma (70\%) o el nervio óptico homolateral $(30 \%)^{35}$.

Antes de ingresar en la cisura interhemisférica se anastomosa a su homóloga contralateral por intermedio de la arteria comunicante anterior. Se ubica luego en relación al cuerpo calloso al que contornea, para terminar en su rodete ${ }^{24,32,39,40,42,48,50}$.

En este trayecto se reconocen 5 sectores: $\mathrm{Al}$ o precomunicante, A2 o subcalloso, ubicado por debajo de la rodilla del cuerpo calloso; A3, precalloso; A4 o supracalloso y A5, postcalloso $\mathrm{og}^{29,35,40}$. La ACA distal corresponde a los sectores A2-A5.

Algunos autores consideran como arteria pericallosa (APC) al ramo que surge luego de que la ACA origina a la arteria callosomarginal (ACM) ${ }^{39}$. Dada la variabilidad en morfología, topografía y calibre de dicha bifurcación, Rhoton ${ }^{39-42}$ expresa que la arteria cerebral anterior, a partir de la comunicante anterior, debe denominarse arteria pericallosa y la arteria callosomarginal sería un ramo de la misma. El punto de origen y calibre de dicha arteria es variable.

La arteria pericallosa da varios ramos colaterales corticales como las arterias órbitofrontal, frontopolar, callosomarginal, frontales internas anterior, media y posterior, parietales superior e inferior y paracentral. Dentro de este esquema, se reconocen variaciones entre las cuales se destaca la presencia de una arteria callosomarginal gruesa, que puede confundirse con la pericallosa ${ }^{50}$. De la misma forma, alguna de las arterias frontales puede originarse de la callosomarginal.

Además de dar estos ramos, la APC nutre el cuerpo calloso y da pequeñas perforantes basales para hipotálamo, septum pellucidum, fornix, comisura blanca anterior y porción anteroinferior del cuerpo estriado ${ }^{24,25}$. En más del $70 \%$ de los casos, la arteria de Heubner nace del sector A2, a nivel yuxtacomunicante ${ }^{41}$. 
Por intermedio de sus ramas, la arteria pericallosa tiene un territorio de irrigación cortical que abarca: la mayor parte de la cara interna del hemisferio cerebral llegando hasta el surco parietooccipital, la mitad interna de la cara basal del lóbulo frontal, la cara externa de la primera circunvolución frontal (F1) y el sector superior de las áreas pre y postcentral ${ }^{32,50,56}$. Aporta además la vascularización para el cuerpo calloso y en $2 / 3$ de los casos, una pericallosa da ramos para el hemisferio contralateral ${ }^{25,40,41}$.

Entre las principales variaciones de la arteria pericallosa, se destacan la arteria mediana del cuerpo calloso, la arteria ácigos cerebral anterior, la pericallosa bihemisférica ${ }^{3,30,41}$. En esta última variante, una de las pericallosas tiene una importante participación en la vascularización del hemisferio contralateral ${ }^{3}$. Las variaciones mencionadas, en su conjunto representan más del $10 \%$ de los casos ${ }^{30}$.

Rhoton plantea que la ubicación de un aneurisma se basa en 4 principios anatómicos: 1) se originan en una bifurcación arterial, 2) nacen en relación a una curva de la arteria madre, 3) el fondo apunta hacia donde se dirigiría el flujo sanguíneo en caso de no existir una bifurcación o una curva, 4) cerca de cada topografía aneurismática nacen arterias que deben protegerse. Según estas reglas, los aneurismas de la arteria pericallosa pueden ubicarse en el origen de cualquiera de sus ramas corticales, aunque en realidad predominan en la bifurcación arterial de mayor flujo (arterias pericallosa-callosomarginal).

\section{Características de la población portadora}

En la mayoría de las series consultadas, la edad promedio de los pacientes va desde los 40.6 a 54 años , $17,27,34,41,56$ lo que concuerda con esta presentación. Las edades estuvieron comprendidas entre los 34 y 69 años, con un promedio de 48.6.

En los pacientes analizados, predominaron los hombres en una relación de 5:1, a diferencia de lo que marcan la mayoría de los trabajos consultados, donde predominan las mujeres en proporción variable: de 1.2:1 a 2:1,17,26,34. Sólo en las series de Laitinen ${ }^{27}$, Yasargil ${ }^{60}$ y Hernesniemi ${ }^{18}$ hay predominancia masculina.

Entre los antecedentes personales que tienen incidencia para la $\mathrm{HSA}^{4,47}$, se hallaron: 5 casos HTA, 4 individuos tabaquistas, alcoholismo y obesidad en una oportunidad respectivamente.

Un paciente (caso 2) tenía cargados antecedentes familiares de enfermedades cerebrovasculares: un hermano portador de una MAV y un primo fallecido por HSA. Otro paciente tenía un hermano fallecido por HSA. Si bien ambos pacientes cumplen con los criterios para ser considerado como familia portadora de aneurismas, no se estudió al resto de los integrantes de la misma ${ }^{25}$.

La incidencia de aneurismas de presentación familiar es de $7 \%{ }^{16}$; en la serie aquí reportada, representaron un
$11.2 \%$. Característicamente, se describe que estos pacientes sufren HSA a edades más tempranas que la población genera ${ }^{16}$. Nuestros hallazgos son coincidentes, ya que los dos pacientes con antecedentes familiares están por debajo del promedio de edad de la serie y uno de ellos es el más joven del grupo estudiado. Uno de los sujetos con antecedentes familiares de HSA, fue portador de un aneurisma fusiforme.

\section{Presentación clínica}

El cuadro clínico típico de HSA, con una cefalea explosiva, diferente a todas las que ha tenido el enfermo antes, se presentó en 11 de 13 casos. Esto es hallado por otros autores en un porcentaje que va del 50 al $100 \% \%^{9,20,28,42}$. Para Solomon y $\mathrm{Awad}^{49}$, los síntomas o signos que le siguen en frecuencia a la cefalea, son las náuseas o vómitos, rigidez de nuca y pérdida de conocimiento, lo cuál concuerda con los hallazgos relatados.

Como forma de presentación poco frecuente, se encuentran las crisis generalizadas ${ }^{10}$. En esta serie, un paciente tuvo una crisis generalizada sin otros síntomas. Otro, presentó una crisis generalizada y posteriormente cefaleas. Dos pacientes tuvieron crisis en la evolución, pero éste no fue el debut del cuadro. La presentación sólo con crisis es poco frecuente: $6.9 \%$ en 72 pacientes según De Souza ${ }^{9}$; ningún caso en 10 pacientes de Inci ${ }^{20}$.

Si bien no hay síntomas típicos que definan la topografía en la arteria pericallosa, se citan algunos elementos orientadores a un compromiso del sector distal de la arteria cerebral anterior. Entre ellos se encuentran: paraparesia o monoparesia crural contralateral al aneurisma, hemiparesia de predominio crural, hipoestesia a nivel del miembro inferior parético, por compromiso del lóbulo paracentral; ya excepcionalmente incontinencia esfinteriana o trastornos conductuales secundarios a disfunción del lóbulo frontal, afasia transitoria por caída de flujo en la pericallosa izquierda o mutismo aquinético $0^{4,7,27,43,58}$. Este último se asocia en general a un hematoma frontal ${ }^{20}$. Sin embargo, la única paciente de esta serie que se presentó con un hematoma frontal, no tuvo aquella presentación clínica.

Se debe mencionar que ninguno de los pacientes sufrió episodios clínicos que hagan pensar en un resangrado, si bien Sindou ${ }^{44}$ plantea que el índice de dicha complicación en estos aneurismas es alta $16.7 \%$ contra $5 \%$ en el resto de las topografías.

Tampoco se vieron pacientes en edad pediátrica $o$ aneurismas de origen traumático, como citan otros auto$\operatorname{res}^{8,10,62}$.

\section{Hallazgos tomográficos}

En 10 de 13 de los casos, la TAC evidenció sangre a 
nivel cisternal y fue diagnóstica de HSA. En dos pacientes, se encontró respectivamente un hematoma frontal y un área de isquemia frontal anterior. Dos enfermos tuvieron una TAC normal y se diagnosticaron mediante punción lumbar. Esto es particularmente importante de señalar: en estos individuos (casi el 17\% de esta serie) fue la sospecha clínica lo que llevó al planteo de HSA, a pesar de que la TAC fue normal.

En cuanto a la distribución de la sangre en el espacio subaracnoideo, Fisher ${ }^{12}$ plantea que en la mayoría de los sujetos, la distribución del sangrado es orientadora de la topografía del aneurisma. Este autor expresa que orienta al sangrado de un aneurisma pericalloso lo siguiente: 1) la presencia de sangre en la porción anterior de la cisterna pericallosa y 2) hematomas del cuerpo calloso. La HSA en la cisterna interhemisférica puede verse también en relación a un aneurisma de la arteria comunicante anterior, por de más bien frecuentes ${ }^{15,33}$.

Otros hallazgos tomográficos que se citan en relación a la HSA por AAPC son: HSA difusa, hematoma frontal, hematoma subdural agudo interhemisférico y hemorragia intraventricular.

Por otra parte, para Auer ${ }^{2}$, la presencia de hematomas intraparenquimatosos e intraventriculares, es un factor que afecta claramente de forma negativa el pronóstico final del paciente, incluso frente a igual grado en las escalas de Hunt y Hess y la WFNS ${ }^{28}$.

\section{Situación y morfología de los aneurismas en la ACAD}

Si bien se describen aneurismas en cualquier sector de la arteria pericallosa, la mayoría se sitúan en el origen de la arteria callosomarginal ${ }^{4,8,29,53,55,58}$. El porcentaje de aneurismas pericallosos de esta topografía es variable según las series: 41.2 a $82.4 \% \%^{1,10,11,19,36,62}$. Las otras localizaciones que se describen son menos frecuentes: 1) proximales en el origen de las arterias frontopolar o frontoorbitaria, 2) distales en la terminación de la pericallosa, 3) periféricos en las ramas de la pericallosa o la callosomarginal ${ }^{1,2,29,63}$.

Desde el punto de vista quirúrgico, $\mathrm{Ohno}^{31}$ los divide en tres tipos de acuerdo a su origen, ubicación con respecto al cuerpo calloso y abordaje quirúrgico. Este autor reconoce aneurismas infracallosos, de la rodilla y supracallosos. Yoshimoto ${ }^{63}$ los divide en dos grupos: de la porción ascendente de la pericallosa y de la porción horizontal. Como se verá más adelante, ambos grupos tienen abordaje diferente.

En todas las series consultadas, predominan francamente los aneurismas saculares; los fusiformes son hasta el $10 \%$ de los $\operatorname{casos}^{13}$.

Son muy escasos los reportes de aneurismas gigantes de la arteria pericallosa; hasta el año 1992 sólo había publicados $10 \operatorname{casos}^{37}$. Se plantea que esto se debe a la tendencia de los aneurismas de esta topografía a sangrar, aun cuando tienen pequeño tamaño ${ }^{39,43,52}$.

\section{Abordaje y " timing"}

Los aneurismas pericallosos pueden ser tratados por diferentes abordajes quirúrgicos ${ }^{9,54,63}$.

La elección del mismo dependerá: de la ubicación del aneurisma en la arteria pericallosa, de la preferencia y comodidad del cirujano, y de la presencia o no de aneurismas múltiples ${ }^{9,54}$.

El abordaje interhemisférico basal es el preferido por varios autores, dado que permite llegar a la arteria pericallosa en forma rápida y ofrece un fácil control proximal de los vasos ${ }^{9,44,45,50,52,62}$.

Por ejemplo, Yoshimoto ${ }^{63}$ refiere dos tipos de abordaje, según la situación del aneurisma. Para los que se ubican en la porción ascendente de la pericallosa (entre la comunicante anterior y la rodilla del cuerpo calloso), plantea un abordaje bicoronal en piel y una craneotomía bifrontal con ligadura del seno longitudinal si su calibre no es importante. Posteriormente, se diseca a partir de la bifurcación carotídea, para tener control proximal. De Souza ${ }^{9}$ utiliza un abordaje similar, pero con conservación del seno y con una pequeña craneotomía unilateral, situada a derecha, salvo que haya dominancia venosa en este sector. La presencia de venas puente a nivel frontal puede dificultar el acceso, y su preservación es fundamental, ya que el infarto venoso en un cerebro que ha sufrido una HSA, puede aumentar el edema y complicar el abordaje. Sugita ${ }^{51}$ y Sampei ${ }^{43}$ afirman que los pacientes, en quienes se han sacrificado venas frontales en un abordaje interhemisférico, presentan tres veces más complicaciones isquémicas frontales, que aquellos en donde no lo hubo. Por otra parte, describen cuatro venas frontales de importancia: las venas frontopolar y frontales anterior, media y posterior ${ }^{22,33}$.

Otros autores ${ }^{18,38}$ utilizan de forma rutinaria el abordaje pterional para los aneurismas pericallosos bajos, con buenos resultados y sin inconvenientes técnicos. Este abordaje es el utilizado por los autores. Se puede aumentar el área de acceso, ampliando un poco la incisión en piel y el colgajo óseo en casos de aneurismas múltiples y tratables en el mismo acto.

Para los aneurismas de la porción horizontal de la pericallosa, la mayoría de los autores están de acuerdo en utilizar un abordaje en piel en forma de herradura, centrado en la topografía del aneurisma. El colgajo óseo puede ser triangular $^{9,20}$ o cuadrangular ${ }^{18,20,31,37}$, según la preferencia del cirujano, pero su base queda por lo menos en el borde del seno. El "flap" de dura se hace hacia la línea media y se avanza por la cisura interhemisférica conservando en lo posible, las venas puente (a veces es necesario "esqueletizarlas"). Por este abordaje, el cirujano puede encontrarse de entrada con el fondo del aneurisma, lo que tendría un 
mayor riesgo potencial de rotura antes del clipado.

Algunos autores, mencionan como útil la sección de la rodilla del cuerpo calloso para permitir el control proximal de la arteria pericallosa ${ }^{10}$. En ningún caso de esta serie fue necesario recurrir a dicha técnica.

De Souza ${ }^{9}$ recomienda comenzar disecando el sector anterior de la cisterna interhemisférica y dirigirse luego hacia distal para tener dominado el vaso aferente. Otra posible complicación de esta vía, es que el cirujano confunda la circunvolución del cíngulo con el cuerpo calloso y la arteria callosomarginal con la pericallosa. Inci ${ }^{20}$ recomienda la observación cuidadosa de la arteriografía en el preoperatorio para tener en cuenta los calibres vasculares de ambas arterias, y su topografía específica.

Desde un punto de vista anatómico, la principal diferencia entre el cuerpo calloso y la circunvolución del cíngulo, es el color blanco intenso del primero.

Yasargil ${ }^{60}$ reporta como frecuente la rotura intraoperatoria de los aneurismas pericallosos. Esto se debería a la adherencia del saco al gyrus del cíngulo y a que el cirujano se encuentra antes con el fondo, que con el cuello. Sin embargo Houkin ${ }^{19}$, que estudia las causas específicas de la rotura precoz intraoperatoria de los aneurismas en general, no halla una diferencia significativa entre los aneurismas pericallosos y los de otras topografías.

En esta serie, sólo hubo una rotura intraoperatoria, inmediatamente antes de que se clipara. Este hecho no influyó en la evolución postoperatoria del paciente (GOS I).

En cuanto a la oportunidad operatoria, si bien hay varios trabajos que muestran que la incidencia de complicaciones no es mayor en pacientes operados en agudo que en diferido ${ }^{2,28}$, los autores prefieren operar los aneurismas de topografía poco habitual en diferido. Igualmente hay otros factores no dependientes de los cirujanos que influyen en esta decisión, como: captación tardía de pacientes por errores diagnósticos, referencia tardía de pacientes desde centros del interior del país y dificultad para realizar en ciertas circunstancias una arteriografía de los 4 vasos, de urgencia.

Si bien en este trabajo se presentan sólo pacientes operados a cielo abierto, se debe mencionar la posibilidad de abordar los aneurismas pericallosos por vía endovascular, por equipos especialmente entrenados y con experiencia ${ }^{37}$.

\section{Evolución postoperatoria}

Se consideraba clásicamente a los aneurismas ubicados en la APC como de peor pronóstico quirúrgico, en relación a aquellos situados en otras topografías de la circulación anterior $^{17,44,61}$, aunque trabajos recientes muestran mejores resultados ${ }^{28}$.

En la presente serie la mortalidad global fue de 5.5\%.
De los 13 pacientes en los que se pudo hacer seguimiento y análisis completo de la historia clínica, 10 tuvieron una evolución excelente o buena (GOS I y II). Aquellos con GOS II, soportan trastornos neurosíquicos que no les impiden sus tareas habituales.

Estos resultados coinciden con los de autores que encuentran buenos resultados en la cirugía de los aneurismas de la ACAD. Por ejemplo, Yoshimoto ${ }^{62}$ y Takaku ${ }^{53}$ sobre 1000 pacientes operados, tienen una mortalidad global del $6.1 \%$. Dentro del total, la mortalidad más baja se vio en los aneurismas pericallosos (4.1\%). Otros, observan mortalidad alta específicamente en los aneurismas pericallosos, llegando hasta $15 \%^{16,30,43}$. Yasargil ${ }^{59}$ es uno de los que plantea que los APC ofrecen al cirujano dificultades técnicas que inciden en una mayor mortalidad. Sin embargo en su serie de AAPC logra $0 \%$ de mortalidad ${ }^{59}$.

Los resultados excelentes o buenos (GOS I y II), se reportan entre el $50.4 \%$ y el $81.3 \%$ de los $\operatorname{casos}^{43,53,60}$.

En la serie aquí expuesta, 12 de los 13 enfermos desarrolló una buena o excelente evolución. Todos los que tuvieron un GOS I final, ingresaron en el preoperatorio con un score de la escala de Hunt y Hess, de I al III.

Es bien conocido, que entre las secuelas postoperatorias, los trastornos neuropsíquicos son de lo más frecuente en los aneurismas del complejo arterial comunicante anterior y representan cerca de un $10 \%$ de los $\operatorname{casos}^{53}$.

\section{Bibliografía}

1. Afshar, F.: Direct surgical obliteration of frontopolar artery aneurysm. Surg Neurol. 1981; 15: 135-136.

2. Auer, L.M.: Unfavorable outcome following early surgical repair of ruptured cerebral aneurysms. A critical review of 238 patients. Surg Neurol. 1991; 35: 152-158.

3. Baptista, A.G.: Studies of the arteries of the brain II. The anterior cerebral artery: some anatomical features and their clinical implications. Neurology 1963; 13: 825-835.

4. Becker, H., Newton, T.H.: Distal anterior cerebral artery aneurysm. Neurosurgery 1979; 4: 495-503.

5. Bonilha, L., Marques, E.L., Carelli, E.F., Fernandes, Y.B., Cardoso, A.C., Maldaum, M., Borges, G.: Risk factors and outcome in 100 patients with aneurysmal subarachnoid hemorrhage. Arch Neuropsiquiatr. 2002; 59: 676-680.

6. Calvo, A., Mañana, G., Tarigo, A., Spagnuolo, E.: Complicaciones parenquimatosas hemorrágicas de los aneurismas encefálicos. Estudio clínicoanatómico retrospectivo de 23 casos del laboratorio de neuropatología del Instituto de Neurología de Montevideo de 1963 a 1979. Rev. Hosp Maciel 1994; 274-313.

7. Critchley, M.: The anterior cerebral artery, and its syndromes. Brain 1930; 53: 120-165.

8. Crowell, R.M., Qgilvy, C.S.: Anterior communicating artery aneurysms and distal anterior cerebral artery aneurysms. 
En: Tindall, G.T., Cooper, P.R., Barrow, D.L. (Eds.) The Practice of Neurosurgery. Williams and Wilkins. Baltimore, 1996.

9. De Souza, A.A., Dantas, F.L.R., de Cardoso, G.T.C., Costa, B.S.: Distal anterior cerebral artery aneurysms. Surg Neurol 1999; 52: 128-136.

10. Dickey, P.S., Bioomgarden, G.M., Arkins, T.J., Spencer, D.D.: Partial callosal resection for pericallosal aneurysms. Neurosurgery 1999; 30: 136-137.

11. Ferguson, S.M., Rayport, M., Corrie, W.S.: Neuropsychiatric observations on behaviorai consequences of corpus callosum section for seizure control. En: Reeves, AG. (Ed.) Epilepsy and the corpus callosum. Plenum Press. New York. 1985; pp: 501-514.

12. Fisher, R.G., Ciminelo,V.: Perícallosal Aneurysms. J Neurosurg. 1966; 25: 512-515.

13. Geuna, E., Arrigoni, M., Bellotti, C., Formaggio, G.: Les anéurismes de l'artère péricalleuse. Etude critique des problèmes posés et des complications. Neurochirurgie 1979; 25: 19-24.

14. Geschwind, N.: The frequency of callosal syndromes in neurological practice. En: Reeves, AG. (Ed.) Epilepsy and the corpus callosum. Plenum Press. New York. 1985; pp: 349356.

15. Gómez Llata, S., Alvarez Sandoval, D., Gómez Amador, J.L., Mateos Gómez, H.: Aneurismas intracraneales, tratamiento quirúrgico. Arch Neurocien. (Mex) 2002; 7: 6875.

16. Golfinos, J., Zabramski, J.M.: The genetics of intracranial vascular malformations. En: Raffel, C., Harsh, G.R., IV (Eds.) The molecular basis of neurosurgical disease. Concepts in Neurosurgery Vol 8. Wílliams \& Wilkins, Baltimore, 1996, pp 270-277.

17. Habib, M.: Syndromes de déconnexion calleuse et organisation fonctionelle du corps calleux chez I'adulte. Neurochirurgie. 1998; 44(suppl 1): 102-109.

18. Herniesniemi, J., Tapaninaho, A., Vapalathi, M., Niskanen, M., Kari, A., Luukkonen, M.: Saccular aneurysm of the distal anterior cerebral artery and its branches. Neurosurgery 1992; 31: 994-995.

19. Houkin, K., Kuroda, S., Takahashi, A., Takikawa, S., Ishikawa, T., Yoshimoto, Y., Itamato, K.: Intra-operative premature rupture of the cerebral aneurysms. Analysis of the causes and management. Acta Neurochir (Wien). 1999; 141: 1255-1263.

20. Inci, S., Erbengi, A., Ozgen, T.: Aneurysm of the distal anterior cerebral ertery: report of 14 cases and a review of the literature. Surg Neurol. 1998; 50: 130-140.

21. Jackson, A., Fitzgerald, J.B., Hartley, W.J., Leonard, A., Jates, J.: CT appearance of hematomas in the corpus callosum in patients with subaracnoid hemorrhage. Neuroradiology 1993; 35: 420-423 (Abstract).

22. Jan, M., Kakou, M., Velut, S.: Les voies d'abord du corps calleux. Neurochirurgie 1998; 44(supli 1): 133-137.
23. Kakou, M., Destrieux, C., Velut, S.: Microanatomy of the pericallosal arterial romplex. J.Neurosurg. 2000, 93: 667675.

24. Kakou, M., Velut, S., Destrieux, C.: Vascularisation artérielle et veineuse du corps calleux. Neurochirurgie 1998; 44 (Suppl 1), 431-437.

25. Kojima, M., Nagasawa, S., Lee, Y.E., Takeurchi, Y., Tsuda, E., Mabuchi, N.: Asymptomatic familial cerebral aneurysms. Neurosurgery 1998; 43: 776-781.

26. Krayenbühl, H., Yasargil, M.G.: Cerebral angiography. Butterworths. London. 1968.

27. Laintinen, L., Snellman, A.: Aneurysms of the pericallosal artery: A study of 14 cases verified angiographically and treated mainly by direct surgical attack. J Neurosurg. 1960; 17 : 447-458.

28. Le Roux, P.D., Elliott, J.P., Newell, D.W., Grady, M.S., Winn, H.R.: The incidente cof surgical complications is similar in good and poor grade patients undergoing repair of ruptured anterior circulation aneurysms: a retrospective review of 355 patients. Neurosurgery 1996; 38: 887-895.

29. Mann, K.S., Yue, C.P., Wong, G.: Aneurysms of the pericallosal-callosomarginal junction. Surg. Neurol. 1984; 21 : 261-266.

30. Martínez, F., Spagnuolo, E., Calvo, A., Laza, S., Sgarbi, N., Soria, V., Prinzo, H.: Variaciones del sector anterior del polígono de Willis. Correlación anatomo-angiográfica y su implicancia quirúrgica. (arterias: ácigos cerebral anterior, mediana del cuerpo calloso y cerebral media accesoria. Neurocirugía 2004; 15: 578-588.

31. Ohno, K., Monma, S., Suzuki, R., Matsushima, Y., Hirakawa, K.: Saccular aneurysm of the distal anterior cerebral artery. Neurosurgery 1990; 27: 907-913.

32. Ojemann, R. G., Heros, R.C., Crowell, R.M.: Surgical management of cerebrovascular disease. $2^{\text {nd }}$ edition. Williams \& Wilkins. Baltimore. 1988.

33. Oka, K., Rhoton, A.L. Jr., Barry, M., Rodríguez, R.: Microsurgical anatomy of the superficial veins of the cerebrum. Neurosurgery 1985; 17: 711-748.

34. Perese, D.M.: Superficial veins of the brain from a surgical point of view. J. Neurosurgery 1960, 17: 402-412.

35. Perimutter, D., Rhoton, A.L. Jr.: Microsurgical anatomy of the distal anterior cerebral artery. J Neurosurg. 1978, 49: 204-228.

36. Perimutter, D., Rothon, A.L. Jr: Microsurgical anatomy of the anterior cerebral-anterior communicating-recurrent artery complex. J Neurosurg. 1976; 45: 259-272.

37. Preul, M., Tampieri, D., Leblanc, R.: Giant aneurysm of the distal anterior cerebral artery: associate with an anterior communicating artery aneurysm and a dural arteriovenous fistula. Surg Neurol. 1992; 38: 347-352.

38. Proust, F., Toussaint, P., Hannequin, D., Rabenenoina, C., Le Gars, D., Fréger, P.: Outcome in 43 patients with distal anterior cerebral artery aneurysms. Stroke 1997; 28: 2405- 
2409.

39. Rhoton, A.L. Jr.: Anatomy of saccular aneurysms. Surg Neurol. 1980; 14: 59-66.

40. Rhoton, A.L. Jr.: Anatomic foundations of aneurysm surgery. Clin Neurosurg. 1993; 41: 289-324.

41. Rhoton, A.L. Jr.: Aneurysms. Neurosurgery 2002; 51 (Supp 1): S121-S158.

42. Rhoton, A.L. Jr.: Supratentorial arteries. Neurosurgery 2002; 51 (Supp 1): S53-S120.

43. Sampei, T., Yasui, N., Okudera, T., Fukasawa, H.: Anatomic study of anterior frontal cortical veins with special reference to the frontopolar vein. Neurosurgery 1996; 38: 971975.

44. Sindou, M., Pelissou-Guyotat, I., Mertens, P., Keravel, Y., Athayde, A.A.: Pericallosal aneurysms. Surg Neurol. 1988; 30: 434-440.

45. Smith, R.R., Zubkov, Y.N., Tarrasoli, Y.: Cerebral aneurysms. Microvascular and endovascular management. Springer Verlag. New York. 1994.

46. Spagnuolo, E., Calvo Rubai, A.: Patología vascular hemorrágica encefálica. Oficina del Libro. AEM. Montevideo. 1997; pp: 9-20; 26-36; 37-40; 71-80.

47. Spagnuolo, E., Johnston, E., Tarigo, A., Calvo, A.: Factores que inciden en el pronóstico de los pacientes con hemorragia subaracnoidea por aneurisma. Rev. Hosp. Maciel 1995; 25: 15-22.

48. Spetzler, R.F., Koos, W.T., Richling, B., Lamp, J.: Color Atlas of Microneurosurgery. $2^{\text {nd }}$ edition. Vol II. Thieme. New York. 1997.

49. Solomon, R.A.: Surgical treatment of anterior circulation aneurysms. En: Awad, I (Ed.): Current management of cerebral aneurysms. AANS. 1993; pp: 125-158.

50. Stefani, M.A., Marrrone, A.C.H., Severino, A.G., Schneider, F.L.: Importância clínica e cirúrgica dos ramos distais da artéria cerebral anterior. J. Bras. Neurocirurg. 1997; 8:4150 .

51. Sugita, K., Kobayashi, S., Yokoo, A.: Preservation of large bridging veins during brain retraction. Technical note. J. Neurosurg. 1982, 57: 856-858.

52. Sundt, T.M.: Surgical techniques for saccular and giant intracranial aneurysms. Williams \& Wilkins. Baltimore. 1990.

53. Takaku, A., Tanaka, S., Mori, T., Suzuki, J.: Postoperative complications in 1000 cases of intracranial aneurysms. En: Suzuki, J. (Ed.) Cerebral aneurysms. Experiences with
1000 directly operated cases. Neuron Publishing Co. Tokyo. 1979. pp: 633-643.

54. Tew, J.M Jr., Van Loveren, H.R.: Atlas of operative neurosurgery. Aneurysms and arteriovenous malformations. WB Saunders. Philadelphia. 1994.

55. Van der Zwan, A., Hillen, B., Tulleken, C., Dujovny, M., Dragovic, L.: Variability of territories of the major cerebral arteries. J Neurosurg. 1992; 77: 927-940.

56. Villar, A., Spagnuolo, E., Calvo, A., Tarigo, A., Johnston, E., Valerio, J.: Errores diagnósticos en pacientes con hemorragia subaracnoidea. Rev. Hosp Maciel. 1997; 2: $5-10$.

57. Weir, B.: The surgical management of anterior circulation aneurysms. Neurosurg. Quarterly. 1991; 1: 19-28.

58. Weir, B., Disney, L., Karrison, T.: Sizes of ruptured and unruptured aneurysms in relation to their sites and the ages of patients. J Neurosurg 2002; 96: 64-70.

59. Yasargil, M.G.: Microneurosurgery: Microsurgical Anatomy of the Basal Cistems and Vessels of the Brain, Diagnosis Studies, General Operative Techniques and Pathological Considerations of the Intracranial Aneurysms. George Thieme Verlag. Stuttgart. 1984.

60. Yasargil, M.G., Carter, P.: Saccular aneurysm of the distal anterior cerebral artery. J.Neurosurg. 1974; 39: 218223.

61. Yasargil, M.G., Smith Young, P.H., Teddy, P.J.: Microneurosurgery: Technical complications, surgery of intracranial aneurysms. George Thieme Verlag. Stuttgart. 1984.

62. Yoshimoto, T., Uchida, K., Kaneko, U., Kayama, T., Suzuki, J.: An analysis of follow-up resuits of 1000 intracranial saccular aneurysms with definitive surgical treatment. J.Neurosurg. 1979; 50: 152-157.

63. Yoshimoto, T., Uchida, K., Suzuki, J.: Surgical treatment of distal anterior cerebral artery aneurysms. In: Suzuki, J. (Ed.) Cerebral aneurysms. Experiences with 1000 directly operated cases. Neuron Publishing Co. Tokyo. 1979. pp 250 255 .

Martinez, F.; Spagnuolo, E.; Calvo, A.: Aneurismas del sector distal de la arteria cerebral anterior (arteria pericallosa). Neurocirugía 2005; 16: 333-344.

Correspondencia postal: F. Martínez. Servicio de Neurología y Neurocirugía. Hospital Maciel. Dr. Antonio Calvo MSP - ASSE Montevideo, Uruguay. 
Comentario al trabajo: Aneurismas del sector distal de la arteria cerebral anterior (arteria pericallosa) de Martínez y cols.

Los aneurismas de la arteria pericallosa son poco frecuentes como bien comentan los autores del trabajo. En nuestro hospital representan el 3.3\% de los aneurismas tratados en los últimos 10 años, muy similar a la referida en la literatura ${ }^{3,5,6}$.

Los aneurismas distales de la arteria cerebral anterior tienen unas características especiales que incluyen un espacio muy estrecho para el abordaje interhemisférico, densas adherencias entre el aneurisma y el tejido cerebral; además, con frecuencia, el cuello aneurismático es ancho y esclerótico, asociándose a menudo con otras anomalías vasculares y aneurismas múltiples. También, estos aneurismas son propensos a la ruptura durante la disección debido a las densas adherencias del mismo con el parénquima cerebral y a que la cúpula del aneurisma se dirige hacia el cirujano ${ }^{3}$. Por todo ello, los resultados quirúrgicos y/o endovasculares referidos en la literatura ${ }^{1,4,6}$ no reflejan la gravedad de estos aneurismas. Contrariamente, De Sousa et $\mathrm{al}^{2}$ opinan que los aneurismas distales de la arteria cerebral anterior tienen mayor mortalidad y morbilidad que otros aneurismas supratentoriales.

En un periodo de 10 años hemos ingresado 16 pacientes con aneurismas distales de la arteria cerebral anterior. Cinco pacientes $(31 \%)$ tenían aneurismas múltiples. En dos casos (12.5\%) se asociaron a malformaciones arteriovenosas. Todos menos uno ingresaron después de una hemorragia subaracnoidea. Once pacientes fueron. tratados quirúrgicamente y tres fueron embolizados. Fallecieron cuatro pacientes $(25 \%)$.

\section{Bibliografía}

1. Chalif, D.J.: Surgical treatment of anterior cerebral aneurysnis. En Management of cerebral aneurysm. Le Roux P.D, Winn H.R., Newell DW (eds) Philadelphia. Saunders. 2004. pp 763-794.

2. De Sousa, A.A., Dantas, F.L., de Cardoso, G.T, Costa, B.S.: Distal anterior cerebral artery aneurys. Surg Neurol. 1999; 52: 128-35.

3. Kawashima, M., Matsushima, T., Sasaki, T.: Surgical strategy for distal anterior cerebral artery aneurysms: microsurgical anatomy. J Neurosurg. 2003; 99: 517-525.

4. Menovsky, T., van Rooij, W.J., Sluzewski, M., Wijnalda, D.: Coiling of ruptured pericallosal artery aneurysms. Neurosurgery. 2002; 50: 11-4.

5. Molyneux, A., Kerr, R., Stratton, I., Sandercock, P., Clarke, M., Shrimpton, J., Holman, R., et al.: International Subarachnoid Aneurysm. Trial (ISAT) of neurosurgical clipping versus endovascular coiling in 2143 patients with ruptured intracranial aneurysms: a randomised trial. Lancet. 2002; 360: 1267-1274.

6. Vega-Basulto, S.D., Silva-Adán, S., Mosquera-Betancourt, G.: Cirugía de los aneurismas de la arteria cerebral, anterior distal. Neurocirugía $2003 ; 14: 302-308$.

J. Vilalta

Barcelona 